\title{
Does Physical Activity-Based Intervention Improve Systemic Proinflammatory Cytokine Levels in Overweight or Obese Children and Adolescents? Insights from a Meta-Analysis of Randomized Control Trials
}

\author{
Yanshuo Han ${ }^{a, b}$ Yang Liu ${ }^{a}$ Zhongyi Zhao ${ }^{a}$ Shihan Zhen ${ }^{a}$ \\ Jianhua Chen ${ }^{a, c}$ Ning Ding ${ }^{d}$ Yanan Ma ${ }^{a}$ e Deliang Wen ${ }^{a} d$ \\ ${ }^{a}$ Institute of Health Science, China Medical University, Shenyang, PR China; ${ }^{b}$ Department \\ of General Surgery, Shengjing Hospital of China Medical University, Shenyang, PR China; \\ 'No. 2 Orthopedic Department, Shengjing Hospital of China Medical University, Shenyang, \\ PR China; ${ }^{d}$ Research Center of Medical Education, China Medical University, Shenyang, \\ PR China; eSchool of Public Health, China Medical University, Shenyang, PR China
}

\section{Keywords}

Pediatric obesity · Exercise therapy · Adolescent · Exercise physiology · Inflammation mediators · Physical fitness

\begin{abstract}
Objectives: The purpose of this research was to conduct a meta-analysis of the role that physical activity (PA) plays in influencing the critical proinflammatory cytokine levels associated with overweight/obese children and adolescents to explore the effectiveness of exercise intervention within this population. Methods: With searches of the PubMed, EMBASE, and CENTRAL databases, we updated our meta-analysis up to November 2018. The randomized controlled trials (RCT) evaluated the ability of exercise training to increase the following factors in children and/or adolescents classified as obese or overweight: tumor necrosis factor (TNF)- $\alpha$, interleukin (IL)-6, and C-reactive protein (CRP). Results: Eleven RCT comprising 623 children and/or adolescents who were obese or overweight (i.e., 393 with PA and 230 controls) were suitable for use in this study. The meta-analysis showed that PA in general was associated with a significant reduction of CRP levels (mean difference $=-0.45 \mathrm{mg} / \mathrm{L}, p=0.02$ ) in overweight/ obese children and adolescents. Based on 115 overweight and obese youths, this study suggests that PA does not significantly mitigate IL-6 levels (mean difference $=-0.39 \mathrm{pg} / \mathrm{mL}, p=$ 0.08 ), although there was a trend towards a reduction. Additionally, no close connection was observed between PA and TNF- $\alpha$ levels at $0.04 \mathrm{pg} / \mathrm{mL}(p=0.78)$. Moreover, meta-regression
\end{abstract}


Han et al.: Does PA-Based Intervention Improve Systemic Proinflammatory Cytokine Levels in Overweight or Obese Children and Adolescents?

analysis revealed a statistical association between CPR levels and changes in BMI or changes in adiponectin; likewise, IL-6 levels dramatically impacted the effect of exercise on changes in adiponectin. Conclusions: PA was associated with significantly reduced CRP levels, whereas there was no significant association with IL- 6 or TNF- $\alpha$ in overweight/obese children or adolescents; however, there was a trend towards a reduction of IL- 6 .

(C) 2019 The Author(s)

Published by S. Karger AG, Basel

\section{Introduction}

The prevalence of childhood overweight or obesity has increased worldwide and constitutes a major public health crisis that brings about economic consequences and substantial healthcare costs [1-3]. Moreover, childhood obesity is associated with increased comorbidities, such as insulin resistance, diabetes mellitus, hypertension, sleep apnea, and cardiovascular disease [4-6], which also give rise to an increase in both direct and indirect costs [7]. Recently, the NCD Risk Factor Collaboration pooled population-based data from 1975 to 2016 to update the worldwide trends in pediatric obesity and showed that the increase in childhood and adolescent BMI has plateaued in high-income countries. However, some Asian countries have also demonstrated this plateau, albeit at high levels. Such trends are no longer correlated with those in adults [8]. For these reasons, avoiding obesity in childhood and identifying children with obesity and overweight problems at an early age, to begin intervention efficiently and maintain a healthy weight, is challenging and extremely urgent.

Lifestyle interventions for children and adults have included nutrition advice, regular exercise, and/or other weight loss programs. A recent meta-analysis suggested that a very large number of overweight or obese children and adolescents could benefit from lifestyle interventions [9]. Regarding the nutrition protocol, decreasing energy intake and/or increasing calorie expenditure has been adopted as a common method to promote weight loss [10]. Nevertheless, the results of the effects of increased physical exercise on weight still need further discussion, even though physical exercise has been acknowledged by scholars for decades as having beneficial effects, such as long-term protection against cardiovascular disease $[11,12]$. However, physical activity (PA) seems to increase adiponectin levels but decrease resistin levels [13]. In reality, the function of PA in obesity treatment is too unclear for clinical practice [14].

During recent years, this protective impact has been associated with the modulation of inflammatory processes induced by exercise, as the risk of cardiovascular disease and chronic metabolic disease $[12,15]$ can be reduced through regular exercise, partially because antiinflammatory effects are generated by exercise $[16,17]$. There is an association between inflammatory cytokines and obesity in both adults and children, which suggests that there are some key inflammatory markers that have been consistently connected to both obesity and the risk of adverse outcomes in obesity-associated diseases [18]. For adults, chronic PA reduces serum inflammatory cytokine levels through multiple mechanisms, including decreases in proteic production by skeletal muscles [19], blood mononuclear cells [20], adipose tissue [21, 22], and endothelial cells [23], which can improve endothelial function and insulin sensitivity [22, 24]. In adolescents and children, the reported data tentatively suggest the ability of PA to reduce anti-inflammatory and proinflammatory cytokines [2527]. Nevertheless, several studies have suggested that PA does not seem to decrease proinflammatory cytokine levels in overweight or obese children or adolescents [28, 29], although there are trends towards reductions in these cytokine levels in randomized controlled trials (RCT). Interestingly, the publication of results from a study of adolescent Korean girls with obesity shed light on exercise as a more effective method for improving the levels of serum 
Han et al.: Does PA-Based Intervention Improve Systemic Proinflammatory Cytokine Levels in Overweight or Obese Children and Adolescents?

proinflammatory cytokines, such as C-reactive protein (CRP) [30]. Thus, evidence of the effect of exercise on the control of inflammatory cytokine (pro- or anti-inflammatory) levels in overweight or obese children remains unclear.

Therefore, the purpose of this study was to conduct a meta-analysis of the studies on the role PA plays in modulating critical proinflammatory cytokine levels associated with overweight or obesity in children and adolescents to explore the effectiveness of exercise intervention within this population.

\section{Materials and Methods}

This study was undertaken based on the Cochrane Collaboration specified protocol (handbook version 5.1, available at http://handbook-5-1.cochrane.org/) and reported based on the Preferred Reporting Items for Systematic Reviews and Meta-Analyses (PRISMA) statement (available at http://www.prisma-statement.org/) [31].

\section{Literature Search}

A systematic literature search of relevant English articles on PA intervention and maintenance of usual activities (control) of children and adolescents who were overweight or obese was carried out using the MEDLINE database (PubMed) (1966 to November 2018), the EMBASE database (Embase) (1974 to November 2018), and the CENTRAL database (Cochrane Central Register of Controlled Trials) (2002 to issue 11 of 12, November 2018) by 2 independent researchers (Y.H. and Z.Z.). For the MEDLINE database search, the following Medical Subject Heading (MeSH) search terms were used: pediatric obesity, adolescent, exercise, randomized controlled trials as topic, and cytokines (online suppl. material; for all online suppl. material, see www.karger.com/doi/10.1159/000501970). In addition, exploded keywords included teen*, physical exercise*, and inflammatory*. For the EMBASE database search, the terms used were: randomized controlled trial, clinical trials as topic/exp, obesity/ exp, overweight/exp, and PA. The CENTRAL database was explored using the following keywords: randomized controlled trials, clinical trials as topic, obesity, overweight*, PA, and exercise (online suppl. material). The research process was conducted between November 4 and 10, 2018. We limited our search to English language reports on human subjects. The relevant articles retrieved in this study were thoroughly checked in terms of their references. All literature was registered in the ISI Web of Knowledge: Web of Science database (19912018), through which articles being cited can be found.

\section{Selection}

RCT studies were used only if the following inclusion criteria were met: (a) The children and adolescents were classified as overweight or obese; (b) the individual intervention protocol was premeditated PA (without any dietary intervention); and (c) the study contained sufficient data (at least one proinflammatory cytokine) on factors such as hsCRP, IL-2, IL-6, IL-8, and TNF- $\alpha$.

In cases of multiple reports from the same institution, only the published report with the most details was included to prevent data duplication.

Articles were excluded if it they: (a) did not report outcomes in a comparable fashion, such as with comparisons to a lean group; (b) allowed another kind of physical exercise or dietary restriction intervention to be received by the control group; (c) contained insufficient data (no proinflammatory cytokines); or (d) failed to satisfy the standards for unpublished data and abstracts. 
Han et al.: Does PA-Based Intervention Improve Systemic Proinflammatory Cytokine Levels in Overweight or Obese Children and Adolescents?

\section{Assessment of Risk of Bias}

The quality evaluation was conducted by 2 investigators (Y.H. and S.Z.) independently. Trials were included by methodological quality, which was evaluated with the Cochrane Collaboration tool [32]. The method consists of the following items: random sequence generation (selection bias), allocation concealment (selection bias), blinding of participants and personnel (performance bias), incomplete outcome data (attrition bias), and selective reporting (reporting bias). The evaluation of unclear, low- or high-risk of material bias was made for each item according to the Cochrane Collaboration handbook (version 5.1).

\section{Data Abstraction}

After the initial assessment for eligibility, 2 of the authors (Y.H. and Y.L.) independently conducted the data extraction from the primary sources, and they independently reviewed all of the articles. Any differences in results between the 2 authors were resolved by consensus. For each study, we extracted rudimentary information that included: author, date published, PA group protocol (type, frequency, duration, and intensity), proinflammatory cytokines reported, and control group protocol. Moreover, proinflammatory cytokine levels before and after the intervention period were specifically recorded. Indispensably, requests for missing or incomplete data were sent to every corresponding author.

\section{Quantitative Data Synthesis}

First, we calculated the individual changed score in the weighted mean difference with the $95 \%$ CI from pre- to postintervention between groups for proinflammatory cytokine levels using a method described by Morris [33]. Furthermore, the weighted mean difference was calculated as the sum of the difference in mean proinflammatory cytokine levels before and after the intervention between groups (PA vs. control). Finally, we performed a metaanalysis of proinflammatory cytokine levels using the formula for SEM to represent the SD through the square root of the sample size with the aim of pooling data.

\section{Assessment of Heterogeneity}

This study adopted the fixed effects model unless evidence of significant heterogeneity was found. The degree of heterogeneity was assessed using the $I^{2}$ test. $I^{2}$ values of 25, 25-50, and $>50 \%$ were considered to represent small, medium, and large amounts of inconsistency [34].

\section{Publication Bias Assessment}

Publication bias was detected using a funnel plot. The data points were distributed symmetrically and shaped like an inverted $\mathrm{V}$ when no publication bias was present. Furthermore, to evaluate heterogeneity, the data points were used to form the Egger regression asymmetry plot [35], and Egger graphical exploration was used to assess publication bias [36].

\section{Meta-Regression Analysis}

This study also presents an analysis of the heterogeneity between studies. The following covariates, which might impact the association of proinflammatory cytokines and exercise, were used: (a) duration of exercise in each study (weeks); (b) frequency of sessions per week; (c) duration of exercise per session (min); (d) changes in body composition after the intervention; (e) changes in lipoprotein; ( $f$ ) changes in insulin resistance; (g) changes in blood pressure; and (h) changes in adipokines, such as adiponectin. 
Han et al.: Does PA-Based Intervention Improve Systemic Proinflammatory Cytokine

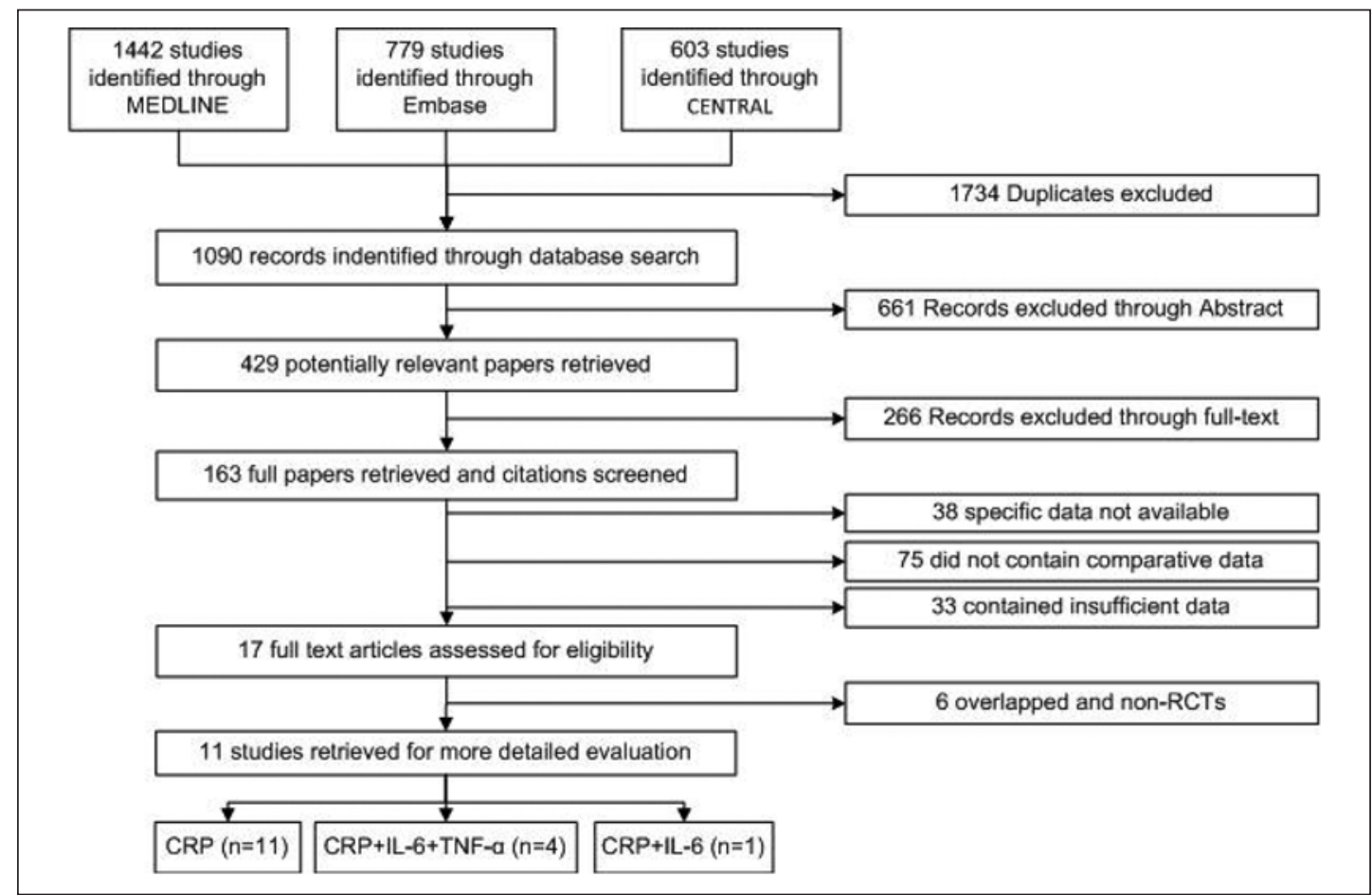

Fig. 1. PRISMA 2010 flow diagram.

\section{Statistical Analysis}

$p<0.05$ was considered statistically significant. A spread sheet was used to conduct all statistical analyses (Microsoft Excel 2010; Microsoft, Redmond, WA, USA), and risk-of-bias assessments, data synthesis, and funnel plots were carried out with Review Manager (version 5.3, available at http://community.cochrane.org/tools/review-production-tools/revman-5/ revman-5-download). Due to statistical software uniqueness and functionality, metaregression and the Egger test/Egger plot were carried out using the meta.ado module of statistical software-STATA (version 12.0; College Station, TX, USA).

\section{Results}

Trial Flow

The literature search identified 163 studies for potential inclusion in the meta-analysis; 146 papers were excluded, 38 studies reported specific data that were not available, 75 did not contain comparative data, and 33 contained insufficient data. Another 17 papers were further assessed for eligibility. Of these, 4 papers overlapped with included studies from the same 2 datasets [37-40] and 4 individuals and did not meet the study design criteria (nonRCT) [41-44]. The trial flow charts for how these were obtained are provided in Figure 1.

\section{Studies Selected}

Table 1 presents the details of the selected articles used in this study. These articles consisted of 11 RCT: 6 independent reports of CRP [28, 30, 45-48], 1 study of CRP and IL-6 [37], and 4 studies of CRP, IL-6, and TNF- $\alpha$ [29, 39, 49, 50]. Two studies included a population of boys only [48, 50], 1 study reported on only girls [30], and 8 studies included both boys 
Han et al.: Does PA-Based Intervention Improve Systemic Proinflammatory Cytokine Levels in Overweight or Obese Children and Adolescents?

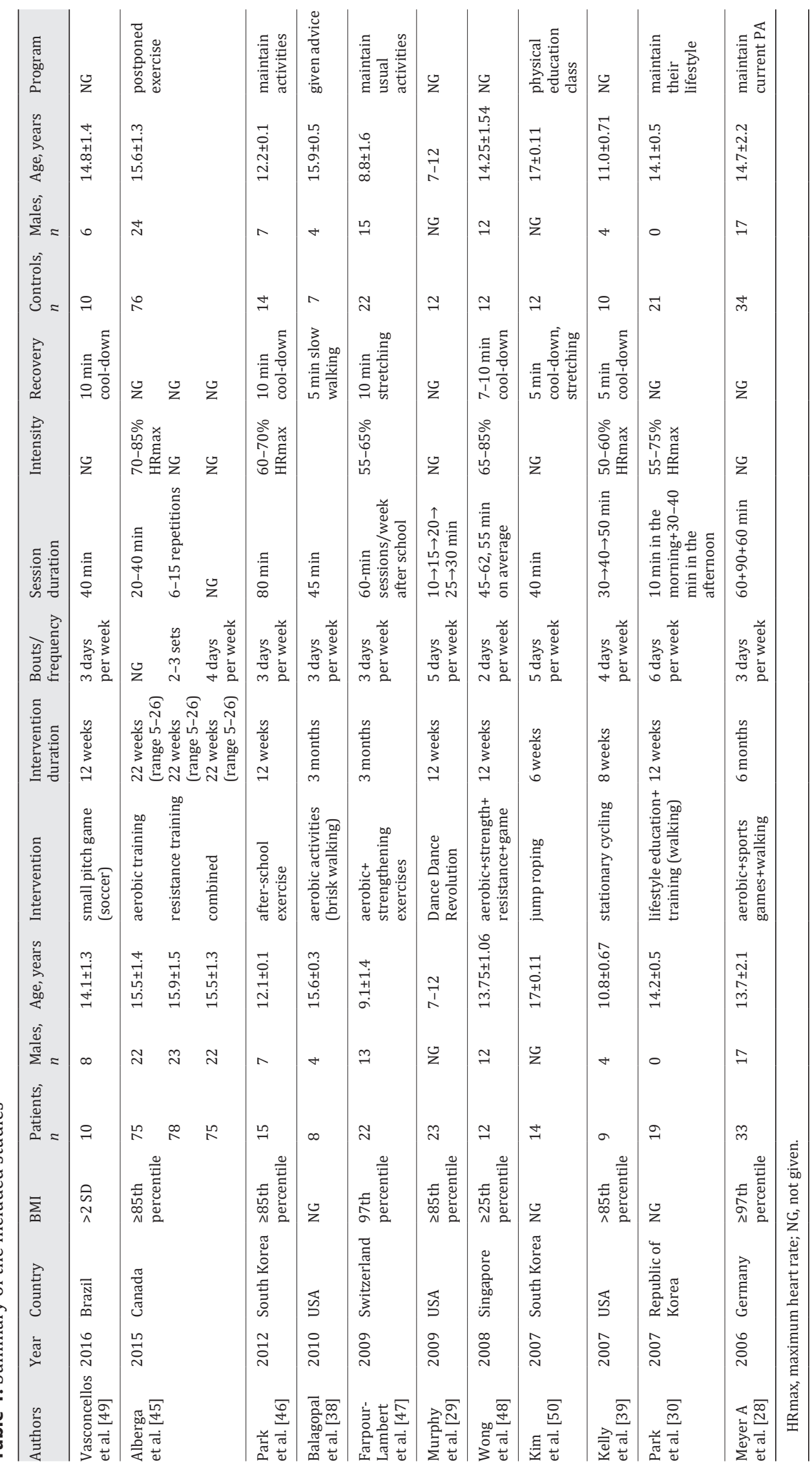


Han et al.: Does PA-Based Intervention Improve Systemic Proinflammatory Cytokine Levels in Overweight or Obese Children and Adolescents?

Table 2. Baseline and postintervention changes in serum CPR level between OE youths and OC youths

\begin{tabular}{|c|c|c|c|c|c|c|c|c|c|c|c|c|c|}
\hline \multirow[t]{2}{*}{ Study } & \multirow[t]{2}{*}{ Year } & \multicolumn{6}{|c|}{ CRP at baseline, $\mathrm{mg} / \mathrm{dL}$} & \multicolumn{6}{|c|}{ CRP postintervention, $\mathrm{mg} / \mathrm{dL}$} \\
\hline & & $\begin{array}{l}\mathrm{OE}, \\
n\end{array}$ & $\begin{array}{l}\mathrm{OE} \\
\text { (mean) }\end{array}$ & $\begin{array}{l}\mathrm{OE} \\
(\mathrm{SD})\end{array}$ & $\begin{array}{l}\text { OC, } \\
n\end{array}$ & $\begin{array}{l}\text { OC } \\
\text { (mean) }\end{array}$ & $\begin{array}{l}\text { OC } \\
\text { (SD) }\end{array}$ & $\begin{array}{l}\text { OE, } \\
n\end{array}$ & $\begin{array}{l}\mathrm{OE} \\
\text { (mean) }\end{array}$ & $\begin{array}{l}\mathrm{OE} \\
(\mathrm{SD})\end{array}$ & $\begin{array}{l}\text { OC, } \\
n\end{array}$ & $\begin{array}{l}\text { OC } \\
\text { (mean) }\end{array}$ & $\begin{array}{l}\mathrm{OC} \\
\text { (SD) }\end{array}$ \\
\hline Vasconcellos et al. [49] & 2016 & 10 & 4.3 & 3.2 & 10 & 3.9 & 1.3 & 10 & 3.7 & 1.8 & 10 & 4.1 & 2.3 \\
\hline Alberga et al. [45] (aerobic) & 2015 & 75 & 2.61 & 2.86 & & & & 55 & 2.23 & 2.37 & & & \\
\hline Alberga et al. [45] (resistance) & 2015 & 77 & 2.64 & 2.89 & 76 & 2.24 & 2.44 & 50 & 2.26 & 2.33 & 56 & 1.98 & 2.10 \\
\hline Alberga et al. [45] (combined) & 2015 & 75 & 1.95 & 2.17 & & & & 58 & 1.9 & 2.06 & & & \\
\hline Park et al. [46] & 2012 & 15 & 1.6 & 1.55 & 14 & 1.9 & 2.62 & 15 & 1.4 & 1.16 & 14 & 0.7 & 0.37 \\
\hline Balagopal et al. [38] & 2010 & 8 & 3.11 & 1.92 & 7 & 3.95 & 3.07 & 8 & 2.22 & 1.47 & 7 & 6.72 & 5.50 \\
\hline Farpour-Lambert et al. [47] & 2009 & 22 & 3.57 & 5.57 & 22 & 4.94 & 4.41 & 22 & 3.43 & 3.67 & 22 & 4.76 & 3.21 \\
\hline Murphy et al. [29] & 2009 & 23 & 3.1 & 2.9 & 12 & 4.7 & 2.7 & 23 & 2.6 & 7.1 & 12 & 4.8 & 2.6 \\
\hline Wong et al. [48] & 2008 & 12 & 3.1 & 1.4 & 12 & 3.4 & 2.4 & 12 & 4.1 & 5.0 & 12 & 4.3 & 3.5 \\
\hline Kelly et al. [39] & 2007 & 9 & 4.4 & 4.8 & 10 & 5.0 & 3.79 & 9 & 4.8 & 7.8 & 10 & 3.8 & 2.85 \\
\hline Kim et al. [50] & 2007 & 14 & 1.7 & 1.87 & 12 & 0.9 & 2.43 & 14 & 1.0 & 2.46 & 12 & 2.1 & 2.43 \\
\hline Park et al. [30] & 2007 & 19 & 1.0 & 1.0 & 21 & 0.8 & 0.5 & 19 & 0.9 & 0.7 & 21 & 1.1 & 0.8 \\
\hline Meyer et al. [28] & 2006 & 33 & 4.84 & 6.31 & 34 & 4.61 & 0.54 & 33 & 2.05 & 2.44 & 34 & 3.36 & 4.76 \\
\hline
\end{tabular}

OE, obesity/overweight exercise; OC, obesity/overweight control.

Fig. 2. Study risk of bias in this meta-analysis.

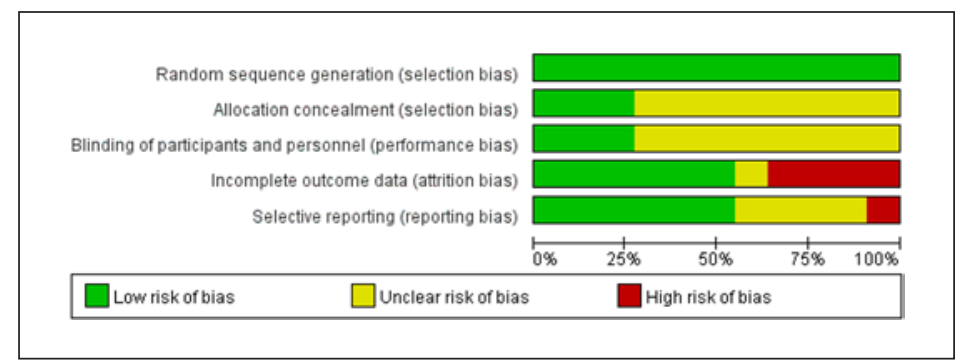

and girls [28, 29, 37, 39, 45-47, 49]. The articles were published from 2005 to 2016 and consisted of 5 from North and South America, 2 from Europe, and 4 from Asia.

\section{Risk of Bias Assessment}

The data (Fig. 2, 3) showed that 3 RCT studies adequately met all of the methodological requirements of the Cochrane risk-of-bias tool; 6 were considered to be at a low risk of bias, and the remaining trial was graded as moderate risk.

\section{Quantitative Data Synthesis}

Main Results

Exercise intervention and CRP in both adolescents and children who suffered from obesity problems were evaluated. The unit of measurement for CRP assessment varied as follows: milligrams/milliliter [50], milligrams/deciliter [46, 49], milligrams/liter [28, 30, 38, $39,45,48]$, micrograms/milliliter [28] and millimoles/liter [47], respectively. Table 2 lists the values for each serum CRP level in all studies before and after the intervention. Overall, PA significantly decreased serum CRP levels ( $n=11$ studies and 430 youths) by $-0.45 \mathrm{mg} / \mathrm{L}$ (95\% CI -0.81 to $\left.-0.08 \mathrm{mg} / \mathrm{L}, p=0.32, I^{2}=13 \%\right)$ in overweight and obese children and adolescents (Fig. 4a). Publication bias was also evaluated with a funnel plot of all studies reporting on the CRP level after exercise, and it demonstrated that all points were within the trilateral region. Interestingly, meta-regression analysis indicated that there was a close association 
Han et al.: Does PA-Based Intervention Improve Systemic Proinflammatory Cytokine Levels in Overweight or Obese Children and Adolescents?

Fig. 3. Reviewers' judgements about each methodological quality item in the included studies. A dot with a plus sign indicates that the item was fulfilled for this study, a dot with a question mark indicates that this item was unclear in this study, and a dot with a minus sign indicates that this study did not fulfil this item.

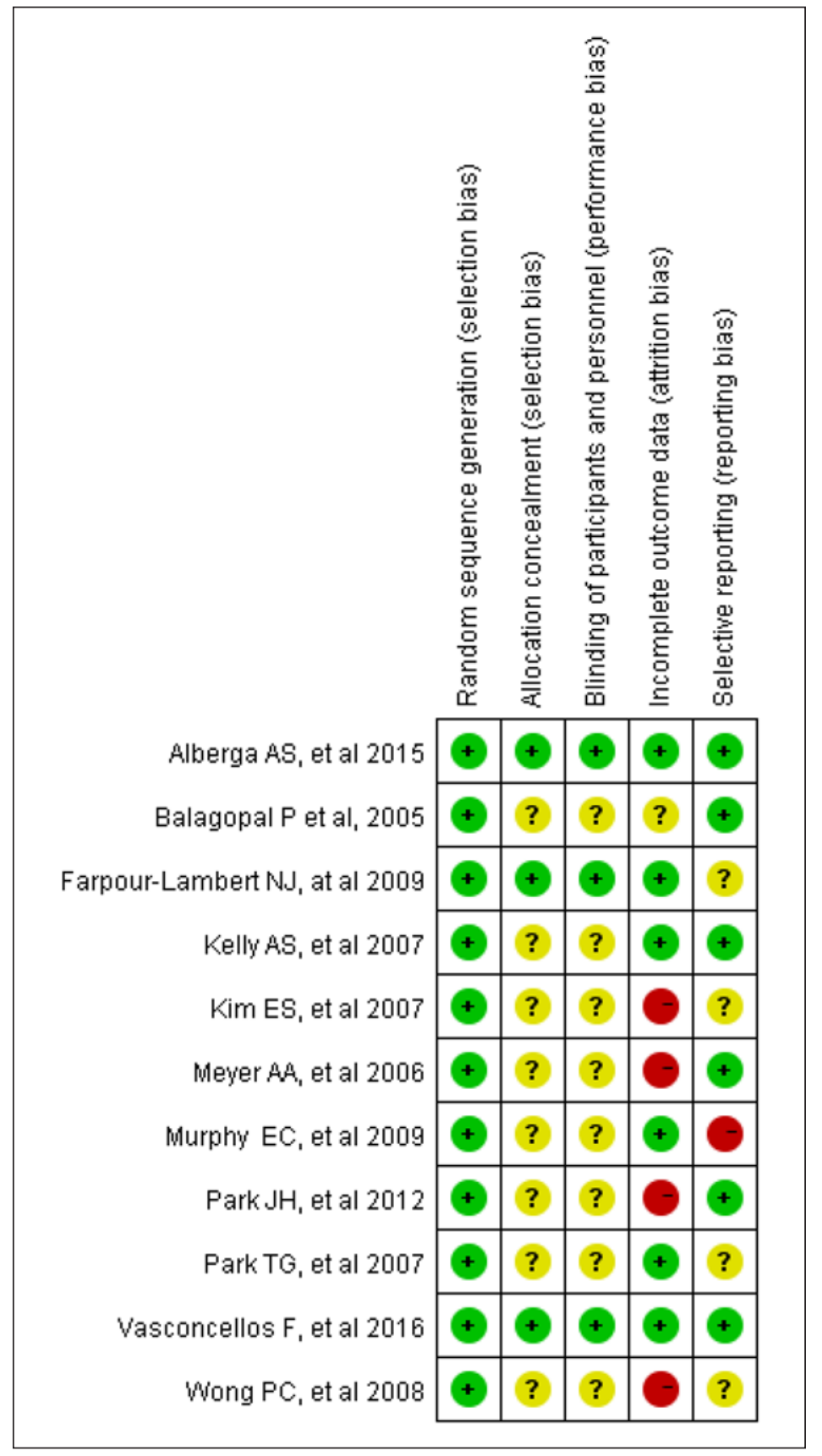

between changes in BMI and CPR levels ( $\beta=0.205$; 95\% CI 0.038-0.421, $p=0.023$ ). Moreover, changes in adiponectin negatively predicted the observed improvements in CRP levels ( $\beta=$ -0.218 ; $95 \% \mathrm{CI}-0.404$ to $-0.029, p=0.0017$ ) but not in the other covariates.

Exercise intervention and IL- 6 in both adolescents and children suffering from obesity problems were evaluated. Of the 11 included trials, 5 RCT analyzed IL- 6 levels, and the unit of measurement varied as follows: picograms/milliliter $[29,39,49,50]$ and milligrams/milliliter [38]. Based on an analysis of 115 youths who were overweight or obese (Table 3), our results suggested that PA did not significantly mitigate the serum IL-6 levels (mean difference $=-0.50 \mathrm{pg} / \mathrm{mL}, 95 \% \mathrm{CI}-1.41$ to $0.40 \mathrm{pg} / \mathrm{mL}, p=0.28, I^{2}=53 \%$ ), although there was a trend toward a reduction (Fig. 4b). Funnel plot asymmetry showed no significant publica- 
Han et al.: Does PA-Based Intervention Improve Systemic Proinflammatory Cytokine Levels in Overweight or Obese Children and Adolescents?

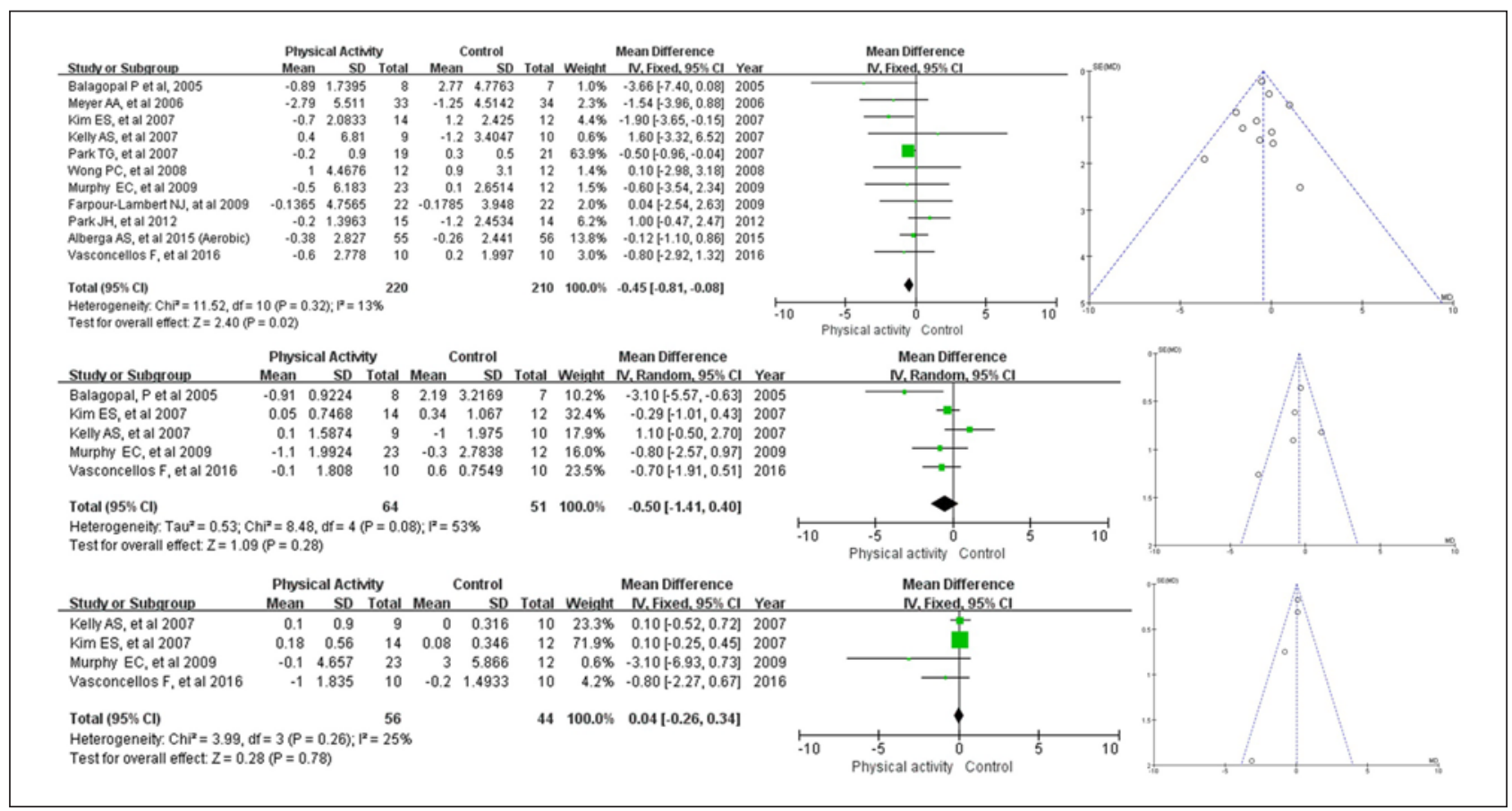

Fig. 4. Absolute changes in CRP (upper panel), IL-6 (middle panel), and TNF- $\alpha$ (bottom panel) in individual studies of the exercise group vs. the control group in obese or overweight children and adolescents.

Table 3. Baseline and postintervention changes in serum IL-6 levels between OE youths and OC youths

\begin{tabular}{|c|c|c|c|c|c|c|c|c|c|c|c|c|c|}
\hline \multirow[t]{2}{*}{ Study } & \multirow[t]{2}{*}{ Year } & \multicolumn{6}{|c|}{ IL-6 at baseline, $\mathrm{pg} / \mathrm{mL}$} & \multicolumn{6}{|c|}{ IL-6 postintervention, pg/mL } \\
\hline & & OE, $n$ & mean & SD & OC, $n$ & mean & SD & $\mathrm{OE}, n$ & mean & SD & OC, $n$ & mean & SD \\
\hline Vasconcellos et al. [49] & 2016 & 10 & 2.2 & 1.7 & 10 & 1.7 & 0.7 & 10 & 2.1 & 1.9 & 10 & 2.3 & 0.8 \\
\hline Murphy et al. [29] & 2009 & 23 & 3.6 & 2.3 & 12 & 4.6 & 3.0 & 23 & 2.5 & 1.2 & 12 & 4.3 & 2.5 \\
\hline Kelly et al. [39] & 2007 & 9 & 2.2 & 1.2 & 10 & 3.5 & 2.2 & 9 & 2.3 & 1.8 & 10 & 2.5 & 1.58 \\
\hline Kim et al. [50] & 2007 & 14 & 0.67 & 0.37 & 12 & 0.53 & 0.17 & 14 & 0.72 & 0.86 & 12 & 0.87 & 1.14 \\
\hline Balagopal et al. [37] & 2005 & 8 & 3.96 & 0.88 & 7 & 4.42 & 1.11 & 8 & 3.05 & 0.96 & 7 & 6.61 & 3.63 \\
\hline
\end{tabular}

OE, obesity/overweight exercise; OC, obesity/overweight control.

Table 4. Baseline and postintervention changes in serum TNF- $\alpha$ level between OE youths and OC youths

\begin{tabular}{|c|c|c|c|c|c|c|c|c|c|c|c|c|c|}
\hline \multirow[t]{2}{*}{ Study } & \multirow[t]{2}{*}{ Year } & \multicolumn{6}{|c|}{ TNF- $\alpha$ at baseline, $\mathrm{pg} / \mathrm{mL}$} & \multicolumn{6}{|c|}{ TNF- $\alpha$ postintervention, $\mathrm{pg} / \mathrm{mL}$} \\
\hline & & OE, $n$ & mean & SD & OC, $n$ & mean & SD & $\mathrm{OE}, n$ & mean & SD & OC, $n$ & mean & SD \\
\hline Vasconcellos et al. [49] & 2016 & 10 & 3.1 & 2.1 & 10 & 2.7 & 1.1 & 10 & 2.1 & 0.8 & 10 & 2.5 & 1.7 \\
\hline Murphy et al. [29] & 2009 & 23 & 11.3 & 4.8 & 12 & 11.1 & 6.1 & 23 & 11.2 & 4.5 & 12 & 11.1 & 6.1 \\
\hline Kelly et al. [39] & 2007 & 9 & 1.3 & 0.3 & 10 & 1 & 0.1 & & 1.4 & 0.3 & 10 & 1 & 0.1 \\
\hline Kim et al. [50] & 2007 & 14 & 1.75 & 0.15 & 12 & 1.68 & 0.1 & 14 & 1.93 & 0.16 & 12 & 1.76 & 0.1 \\
\hline
\end{tabular}

OE, obesity/overweight exercise; OC, obesity/overweight control. 
Han et al.: Does PA-Based Intervention Improve Systemic Proinflammatory Cytokine Levels in Overweight or Obese Children and Adolescents?

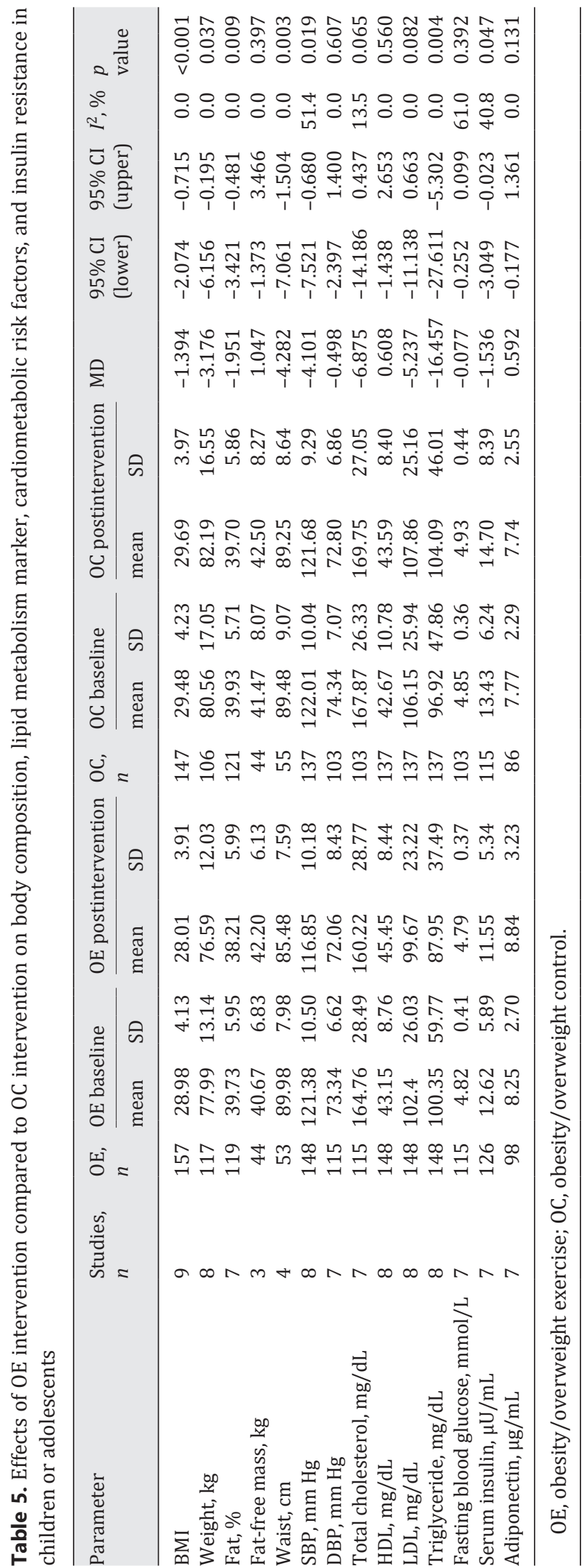


Han et al.: Does PA-Based Intervention Improve Systemic Proinflammatory Cytokine Levels in Overweight or Obese Children and Adolescents?

Table 6. List of publication bias evaluated using a funnel plot and the Egger test

\begin{tabular}{lrlll}
\hline Parameter & Studies, $n$ & Funnel plot & Egger regression intercept & Egger test \\
\hline CRP, mg/L & 11 & - & -0.151 & 0.778 \\
IL-6, pg/mL & 5 & - & -1.174 & 0.541 \\
TNF- $\alpha, \mathrm{pg} / \mathrm{mL}$ & 4 & $?$ & -1.024 & 0.259 \\
BMI & 9 & - & 0.32 & 0.537 \\
Weight, kg & 8 & - & 0.056 & 0.904 \\
Fat, $\%$ & 7 & + & 2.536 & 0.018 \\
Fat-free mass, kg & 3 & $?$ & -0.499 & 0.021 \\
Waist, cm & 4 & - & 0.202 & 0.877 \\
SBP, mm Hg & 8 & - & 0.187 & 0.942 \\
DBP, mm Hg & 7 & $?$ & 1.727 & 0.199 \\
Total cholesterol, mg/dL & 7 & - & 0.303 & 0.891 \\
HDL, mg/dL & 8 & $?$ & 1.286 & 0.104 \\
LDL, mg/dL & 8 & - & 0.935 & 0.551 \\
Triglyceride, mg/dL & 8 & - & -0.976 & 0.362 \\
Fasting blood glucose, mmol/L & 7 & $?$ & -2.017 & 0.293 \\
Serum insulin, $\mu \mathrm{U} / \mathrm{mL}$ & 7 & - & -1.336 & 0.526 \\
Adiponectin, $\mu \mathrm{g} / \mathrm{mL}$ & 7 & - & -0.144 & 0.946 \\
\hline
\end{tabular}

tion bias for a change in IL-6 level. Furthermore, metaregression analysis showed a statistically significant relationship between a change in IL- 6 and a change in adiponectin $(\beta=$ $-0.404 ; 95 \%$ CI -0.709 to $-0.200, p=0.029$ ) but no relationship with the other covariates.

Exercise intervention and TNF- $\alpha$ in both adolescents and children suffering from obesity problems were evaluated. As shown in Table 4, there was no close correlation between PA ( $n=4$ studies and 100 children and adolescents) and TNF- $\alpha$ levels at $0.04 \mathrm{pg} / \mathrm{mL}$ (95\% CI -0.26 to $0.34 \mathrm{pg} / \mathrm{mL}, p=0.78, I^{2}=25 \%$ ), although there was a trend toward an increase (Fig. $4 \mathrm{c}$ ). As the number of studies was limited, we did not perform a meta-regression analysis for TNF- $\alpha$.

\section{Secondary Results}

The results of this meta-analysis revealed a significant mean effect of exercise intervention on reducing the body composition as follows: BMI by -1.394 , weight by $-3.176 \mathrm{~kg}$, percent body fat by -1.951 , and waist circumference by $-4.282 \mathrm{~cm}$. However, the results suggest a nonsignificant trend towards an increase in fat-free mass (mean difference $=1.047 \mathrm{~kg}, p=$ 0.397) following exercise. Significant differences between the PA and the control groups of overweight or obese youths were observed for systolic blood pressure (mean difference = $-4.101 \mathrm{~mm} \mathrm{Hg}$, 95\% CI -7.521 to $-0.680 \mathrm{~mm} \mathrm{Hg}, p=0.019$ ) but not for diastolic blood pressure. Moreover, exercise did not significantly change traditional lipid-related biomarkers, such as total cholesterol, HDL, or LDL, but it did change triglyceride levels (mean difference $=-16.457$ $\mathrm{mg} / \mathrm{dL}, p=0.004$ ). There was also a nonsignificantly greater improvement in fast glucose and serum adiponectin levels, but PA was associated with reductions in fasting insulin levels by $-1.536 \mu \mathrm{U} / \mathrm{mL}(p=0.047)$. All secondary outcomes are summarized in Table 5.

\section{Publication Bias}

First, publication bias was evaluated with a funnel plot of all analyses reporting on dominant results, such as CRP, IL-6, and TNF- $\alpha$, and the secondary results in Table 6 , which demonstrated that most of the points were within the trilateral region. Furthermore, publication bias was evaluated using the Egger test (results in Table 6), and the plot shows that the regression line passes through the point of origin, which may indicate the absence of bias, except for fat percent and fat-free mass. 
Han et al.: Does PA-Based Intervention Improve Systemic Proinflammatory Cytokine Levels in Overweight or Obese Children and Adolescents?

\section{Discussion/Conclusion}

The number of systematic reviews and meta-analyses that have reported, either qualitatively or quantitatively, serum inflammatory cytokine trends in adults suffering from obesity and overweight problems after PA and in obese children or adolescents is limited. To our knowledge, the present study is the first meta-analysis to compare the effectiveness of exercise interventions to that of control interventions for improving proinflammatory cytokines in overweight or obese children and adolescents. In this study, the meta-analysis showed that PA significantly decreased serum CRP levels in overweight or obese children and adolescents. The meta-regression suggested a significant negative relationship between CRP levels and a changes in adiponectin. Moreover, although there was a trend toward a reduction, our results showed no significant association between PA and either TNF- $\alpha$ levels or IL-6 levels.

\section{Statistical Performance of This Meta-Analysis}

For a meta-analysis, heterogeneity is the major concern. However, during this research, there was little evidence of heterogeneity, which partially demonstrated why a well-done randomized controlled study design was adopted. To acquire a higher statistical effectiveness and more accurate conclusions, such as in the study of Alberga et al. [45], we extracted individual serum CRP levels from 3 different exercise groups (aerobic training, resistance training, and combined aerobic and resistance training) as well as from a nonexercising control group. Corresponding data from 3 different exercise intervention groups were analyzed with RevManager and, as expected, the subgroup analysis (online suppl. material) did not change our conclusions. Moreover, because Kelly et al. [40], Kelly et al. [39], Balagopal et al. [37], and Balagopal et al. [38] reported from the same institution, they were treated as one study for the purpose of this analysis. The former data were replenished with the latter and, consequently, all data were fitted into our study.

\section{Five Possible Mechanisms}

Additional adipose tissue underlies the problem of obesity and overweight in children and adolescents. White adipose tissue also functions as a type of endocrine organ that can lead to energy homeostasis [51]. Thus, macrophages that infiltrate white adipose tissue in obesity are responsible for the regulation of the adipose tissue's inflammatory state [18, 52], and the expansion of white adipose tissue leads to the increased generation of proinflammatory adipokines, such as TNF- $\alpha$, leptin, IL-6, and IL-18 [51]. Conversely, the specific number of anti-inflammatory cytokines, such as adiponectin, is decreased [51]. Simultaneously, IL-6 is a powerful inducer of the hepatic acute phase response, which leads to increased concentrations of acute phase reactants, such as CRP $[22,53]$.

The decreased levels of proinflammatory cytokines after exercise intervention in youths may be due to 5 distinct mechanisms. First, PA might improve inflammatory cytokines by reducing the visceral fat mass. Through regular exercise, people can decrease their BMI, fat percentage and waist circumference, with consequently large decreases in abdominal and visceral fat mass; this was also observed in our meta-analysis. Indeed, this meta-regression analysis revealed a statistically significantly positive relationship between the CPR level and a change in BMI. Moreover, exercise might elevate anti-inflammatory cytokines, such as adiponectin. Weight loss improves inflammation in terms of anti-inflammatory cytokines (adiponectin) [51, 54], and this was also observed in our meta-analysis, suggesting a significantly negative relationship between CPR levels and changes in adiponectin by meta-regression. Furthermore, PA may also alter macrophage activation, resulting in an inflammatory cytokine level. M1-type macrophages produce nitric oxide, IL-6, and 


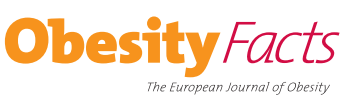

\begin{tabular}{l|l}
\hline Obes Facts 2019;12:653-668 \\
\hline DOI: 10.1159/000501970 & $\begin{array}{l}\text { ○ 2019 The Author(s). Published by S. Karger AG, Basel } \\
\text { www.karger.com/ofa }\end{array}$ \\
\hline
\end{tabular}

Han et al.: Does PA-Based Intervention Improve Systemic Proinflammatory Cytokine Levels in Overweight or Obese Children and Adolescents?

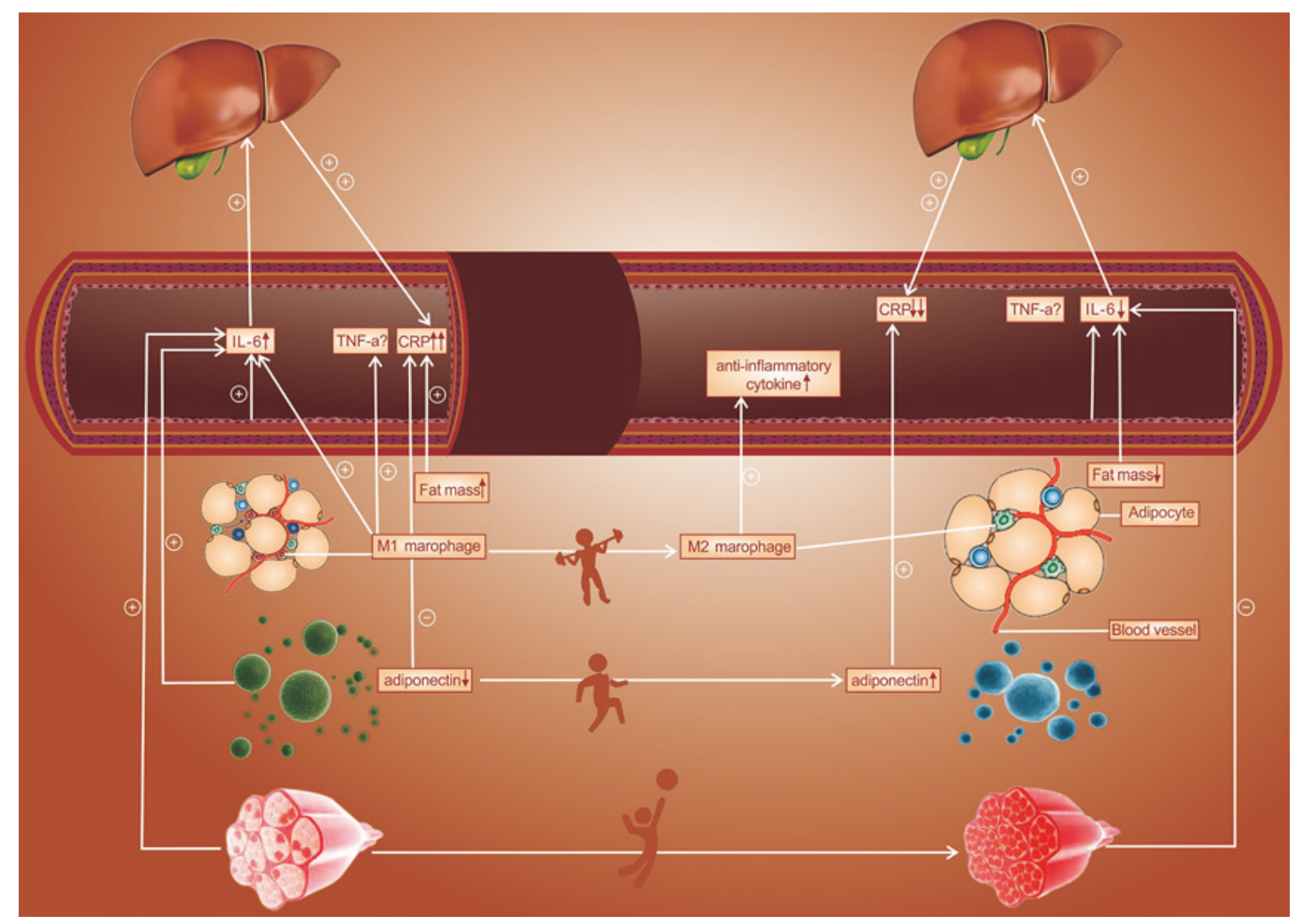

Fig. 5. Potential mechanisms contributing to the anti-inflammatory effects of exercise.

TNF- $\alpha$, while anti-inflammatory cytokines are produced by M2-type macrophages. It is possible that the phenotypic switch from the M1 macrophage to the M2 macrophage is induced by exercise. Additionally, exercise also prevents M1 macrophage infiltration into adipose tissue [55]. Fourth, physical exercise reduces inflammatory cytokines by improving endothelial function. Through regular exercise, obese youth can decrease their systolic blood pressure, and this was verified in our meta-analysis. Endothelial cells are widely known to secrete IL-1 and IL-6; the generation of IL can be increased through activated endothelia and inflammation can then be induced. Therefore, peripheral inflammatory markers associated with endothelial dysfunction are reduced by regular PA [21], as are soluble vascular cell adhesion molecule-1 (sVCAM-1) and macrophage chemoattractant protein 1 (MCP-1) [23]. Finally, exercise may also regulate inflammatory cytokines, particularly IL-6, via muscle contraction. For IL-6 secretion into the systemic circulation, skeletal muscle might serve as an endocrine mechanism. On the one hand, during and after exercise of a sufficient load, active skeletal muscle markedly increases both cellular and circulating levels of IL-6 [56]. On the other hand, a variety of responses in terms of IL-6 levels have been reported; a decrease was observed in some studies [57, 58] and no change was observed in other studies after intervention programs in obese children [59,60]. Thus, several likely mechanisms among those listed above contribute to the anti-inflammatory effect of exercise (Fig. 5).

\section{Limitations}

This meta-analysis has several limitations. First, regarding obesity and overweight, the definitions are inconsistent. Second, a small number of RCT were included, although the stringency of the conclusion criteria already optimized their homogeneity. Furthermore, this meta-analysis 
had heterogeneity, especially for typology of exercises. The dramatic differences in the types of exercise conducted in the interventions may have impacted the CRP levels, Moreover, the metaanalysis was limited to studies in English, so publication bias may have occurred.

\section{Conclusion}

Our study indicated that PA was associated with reduced serum CRP levels, whereas associations with IL- 6 and TNF- $\alpha$ in overweight or obese children or adolescents were nonsignificant, although there was a trend toward a reduction in IL-6. This meta-analysis supports the notion that PA can be used as a therapy for children and adolescents to reverse the lowgrade inflammatory state of overweight/obesity, thereby restoring anti-inflammatory levels to those observed in normal-weight youths. In addition, subsequent studies must delineate the mechanisms through which the inflammatory process is affected by exercise.

\section{Acknowledgement}

The authors would like to thank Nan Jiang for contributing to the final draft of this paper.

\section{Statement of Ethics}

The authors have no ethical conflicts to disclose.

\section{Disclosure Statement}

The authors have no conflict of interests to declare.

\section{Funding Sources}

This work was supported by the China Postdoctoral Science Foundation (grant 2018M640270), the National Science Foundation of China (grant 71774173 and grant 81600370), and the Natural Science Foundation of Liaoning Province of China (grant 20180530007).

\section{References}

1 Pelone F, Specchia ML, Veneziano MA, Capizzi S, Bucci S, Mancuso A, et al. Economic impact of childhood obesity on health systems: a systematic review. Obes Rev. 2012 May;13(5):431-40.

2 Wang Y, Beydoun MA, Liang L, Caballero B, Kumanyika SK. Will all Americans become overweight or obese? estimating the progression and cost of the US obesity epidemic. Obesity (Silver Spring). 2008 0ct;16(10):2323-30.

3 Nasreddine L, Naja F, Akl C, Chamieh MC, Karam S, Sibai AM, et al. Dietary, lifestyle and socio-economic correlates of overweight, obesity and central adiposity in Lebanese children and adolescents. Nutrients. 2014 Mar;6(3):1038-62.

4 Koren D, Chirinos JA, Katz LE, Mohler ER, Gallagher PR, Mitchell GF, et al. Interrelationships between obesity, obstructive sleep apnea syndrome and cardiovascular risk in obese adolescents. Int J Obes. 2015 Jul;39(7):1086-93.

5 Nicholas LM, Morrison JL, Rattanatray L, Zhang S, Ozanne SE, McMillen IC. The early origins of obesity and insulin resistance: timing, programming and mechanisms. Int J Obes. 2016 Feb; 40(2):229-38.

6 Wells JC, Cole TJ. Height, adiposity and hormonal cardiovascular risk markers in childhood: how to partition the associations? Int J Obes. 2014 Jul;38(7):930-5. 
Han et al.: Does PA-Based Intervention Improve Systemic Proinflammatory Cytokine

Levels in Overweight or Obese Children and Adolescents?

7 Jones-Smith JC, Gordon-Larsen P, Siddiqi A, Popkin BM. Is the burden of overweight shifting to the poor across the globe? Time trends among women in 39 low- and middle-income countries (1991-2008). Int J Obes. 2012 Aug;36(8):1114-20.

8 Collaboration NC; NCD Risk Factor Collaboration (NCD-RisC). Worldwide trends in body-mass index, underweight, overweight, and obesity from 1975 to 2016: a pooled analysis of 2416 population-based measurement studies in 128.9 million children, adolescents, and adults. Lancet. 2017 Dec;390(10113):2627-42.

9 Martin A, Saunders DH, Shenkin SD, Sproule J. Lifestyle intervention for improving school achievement in overweight or obese children and adolescents. Cochrane Database Syst Rev. 2014 Mar;(3):CD009728.

10 Spiegelman BM, Flier JS. Obesity and the regulation of energy balance. Cell. 2001 Feb; 104(4):531-43.

11 Medlow P, McEneny J, Murphy MH, Trinick T, Duly E, Davison GW. Exercise training protects the LDL I subfraction from oxidation susceptibility in an aged human population. Atherosclerosis. 2015 Apr;239(2): 516-22.

12 Shern-Brewer R, Santanam N, Wetzstein C, White-Welkley J, Parthasarathy S. Exercise and cardiovascular disease: a new perspective. Arterioscler Thromb Vasc Biol. 1998 Jul;18(7):1181-7.

13 Jamurtas AZ, Stavropoulos-Kalinoglou A, Koutsias S, Koutedakis Y, Fatouros I. Adiponectin, Resistin, and Visfatin in Childhood Obesity and Exercise. Pediatr Exerc Sci. 2015 Nov;27(4):454-62.

14 Chin SH, Kahathuduwa CN, Binks M. Physical activity and obesity: what we know and what we need to know. Obes Rev. 2016 Dec;17(12):1226-44.

15 Warren TY, Barry V, Hooker SP, Sui X, Church TS, Blair SN. Sedentary behaviors increase risk of cardiovascular disease mortality in men. Med Sci Sports Exerc. 2010 May;42(5):879-85.

16 Kasapis C, Thompson PD. The effects of physical activity on serum C-reactive protein and inflammatory markers: a systematic review. J Am Coll Cardiol. 2005 May;45(10):1563-9.

17 Mathur N, Pedersen BK. Exercise as a mean to control low-grade systemic inflammation. Mediators Inflamm. 2008;2008:109502.

18 Choi J, Joseph L, Pilote L. Obesity and C-reactive protein in various populations: a systematic review and metaanalysis. Obes Rev. 2013 Mar;14(3):232-44.

19 Gielen S, Adams V, Möbius-Winkler S, Linke A, Erbs S, Yu J, et al. Anti-inflammatory effects of exercise training in the skeletal muscle of patients with chronic heart failure. J Am Coll Cardiol. 2003 Sep;42(5):861-8.

20 Smith JK, Dykes R, Douglas JE, Krishnaswamy G, Berk S. Long-term exercise and atherogenic activity of blood mononuclear cells in persons at risk of developing ischemic heart disease. JAMA. 1999 May;281(18):1722-7.

21 Tomaszewski M, Charchar FJ, Przybycin M, Crawford L, Wallace AM, Gosek K, et al. Strikingly low circulating CRP concentrations in ultramarathon runners independent of markers of adiposity: how low can you go? Arterioscler Thromb Vasc Biol. 2003 Sep;23(9):1640-4.

22 Yudkin JS, Stehouwer CD, Emeis JJ, Coppack SW. C-reactive protein in healthy subjects: associations with obesity, insulin resistance, and endothelial dysfunction: a potential role for cytokines originating from adipose tissue? Arterioscler Thromb Vasc Biol. 1999 Apr;19(4):972-8.

23 Adamopoulos S, Parissis J, Kroupis C, Georgiadis M, Karatzas D, Karavolias G, et al. Physical training reduces peripheral markers of inflammation in patients with chronic heart failure. Eur Heart J. 2001 May;22(9):791-7.

24 Mayer-Davis EJ, D’Agostino R Jr, Karter AJ, Haffner SM, Rewers MJ, Saad M, et al. Intensity and amount of physical activity in relation to insulin sensitivity: the Insulin Resistance Atherosclerosis Study. JAMA. 1998 Mar;279(9):669-74.

25 Isasi CR, Deckelbaum RJ, Tracy RP, Starc TJ, Berglund L, Shea S. Physical fitness and C-reactive protein level in children and young adults: the Columbia University BioMarkers Study. Pediatrics. 2003 Feb;111(2):332-8.

26 Huang T, Larsen KT, Møller NC, Ried-Larsen M, Frandsen U, Andersen LB. Effects of a multi-component campbased intervention on inflammatory markers and adipokines in children: a randomized controlled trial. Prev Med. 2015 Dec;81:367-72.

27 Nemet D, Eliakim A. Growth hormone-insulin-like growth factor-1 and inflammatory response to a single exercise bout in children and adolescents. Med Sport Sci. 2010;55:141-55.

28 Meyer AA, Kundt G, Lenschow U, Schuff-Werner P, Kienast W. Improvement of early vascular changes and cardiovascular risk factors in obese children after a six-month exercise program. J Am Coll Cardiol. 2006 Nov; 48(9):1865-70.

29 Murphy EC, Carson L, Neal W, Baylis C, Donley D, Yeater R. Effects of an exercise intervention using Dance Dance Revolution on endothelial function and other risk factors in overweight children. Int J Pediatr Obes. 2009;4(4):205-14.

30 Park TG, Hong HR, Lee J, Kang HS. Lifestyle plus exercise intervention improves metabolic syndrome markers without change in adiponectin in obese girls. Ann Nutr Metab. 2007;51(3):197-203.

31 Moher D, Liberati A, Tetzlaff J, Altman DG; PRISMA Group. Preferred reporting items for systematic reviews and meta-analyses: the PRISMA statement. Int J Surg. 2010;8(5):336-41.

32 Higgins JP, Altman DG, Gøtzsche PC, Jüni P, Moher D, Oxman AD, et al.; Cochrane Bias Methods Group; Cochrane Statistical Methods Group. The Cochrane Collaboration's tool for assessing risk of bias in randomised trials. BMJ. 2011 Oct;343(2):d5928.

33 Morris SB; Estimating Effect Sizes From Pretest-Posttest-Control Group Designs. Estimating Effect Sizes From Pretest-Posttest-Control Group Designs. Organ Res Methods. 2008;11(2):364-86.

34 Higgins JP, Thompson SG. Quantifying heterogeneity in a meta-analysis. Stat Med. 2002 Jun;21(11):1539-58.

35 Egger M, Smith GD. Misleading meta-analysis. BMJ. 1995 Sep;311(7007):753-4. 
Han et al.: Does PA-Based Intervention Improve Systemic Proinflammatory Cytokine

Levels in Overweight or Obese Children and Adolescents?

36 Egger M, Davey Smith G, Schneider M, Minder C. Bias in meta-analysis detected by a simple, graphical test. BMJ. 1997 Sep;315(7109):629-34.

37 Balagopal P, George D, Yarandi H, Funanage V, Bayne E. Reversal of obesity-related hypoadiponectinemia by lifestyle intervention: a controlled, randomized study in obese adolescents. J Clin Endocrinol Metab. 2005 Nov; 90(11):6192-7.

38 Balagopal PB, Gidding SS, Buckloh LM, Yarandi HN, Sylvester JE, George DE, et al. Changes in circulating satiety hormones in obese children: a randomized controlled physical activity-based intervention study. Obesity (Silver Spring). 2010 Sep;18(9):1747-53.

39 Kelly AS, Steinberger J, Olson TP, Dengel DR. In the absence of weight loss, exercise training does not improve adipokines or oxidative stress in overweight children. Metabolism. 2007 Jul;56(7):1005-9.

40 Kelly AS, Wetzsteon RJ, Kaiser DR, Steinberger J, Bank AJ, Dengel DR. Inflammation, insulin, and endothelial function in overweight children and adolescents: the role of exercise. J Pediatr. 2004 Dec;145(6):731-6.

41 Lopes WA, Leite N, da Silva LR, Brunelli DT, Gáspari AF, Radominski RB, et al. Effects of 12 weeks of combined training without caloric restriction on inflammatory markers in overweight girls. J Sports Sci. 2016 Oct; 34(20):1902-12.

42 Nascimento H, Alves AI, Medeiros AF, Coimbra S, Catarino C, Bronze-da-Rocha E, et al. Impact of a SchoolBased Intervention Protocol - ACORDA Project - On Adipokines in An Overweight and Obese Pediatric Population. Pediatr Exerc Sci. 2016 Aug;28(3):407-16.

43 Seabra A, Katzmarzyk P, Carvalho MJ, Seabra A, Coelho-E-Silva M, Abreu S, et al. Effects of 6-month soccer and traditional physical activity programmes on body composition, cardiometabolic risk factors, inflammatory, oxidative stress markers and cardiorespiratory fitness in obese boys. J Sports Sci. 2016 0ct;34(19):1822-9.

44 Woo J, Shin KO, Yoo JH, Park S, Kang S. The effects of detraining on blood adipokines and antioxidant enzyme in Korean overweight children. Eur J Pediatr. 2012 Feb;171(2):235-43.

45 Alberga AS, Prud'homme D, Kenny GP, Goldfield GS, Hadjiyannakis S, Gougeon R, et al. Effects of aerobic and resistance training on abdominal fat, apolipoproteins and high-sensitivity C-reactive protein in adolescents with obesity: the HEARTY randomized clinical trial. Int J Obes. 2015 Oct;39(10):1494-500.

46 Park JH, Miyashita M, Kwon YC, Park HT, Kim EH, Park JK, et al. A 12-week after-school physical activity programme improves endothelial cell function in overweight and obese children: a randomised controlled study. BMC Pediatr. 2012 Jul;12(1):111.

47 Farpour-Lambert NJ, Aggoun Y, Marchand LM, Martin XE, Herrmann FR, Beghetti M. Physical activity reduces systemic blood pressure and improves early markers of atherosclerosis in pre-pubertal obese children. J Am Coll Cardiol. 2009 Dec;54(25):2396-406.

48 Wong PC, Chia MY, Tsou IY, Wansaicheong GK, Tan B, Wang JC, et al. Effects of a 12-week exercise training programme on aerobic fitness, body composition, blood lipids and C-reactive protein in adolescents with obesity. Ann Acad Med Singapore. 2008 Apr;37(4):286-93.

49 Vasconcellos F, Seabra A, Cunha F, Montenegro R, Penha J, Bouskela E, et al. Health markers in obese adolescents improved by a 12-week recreational soccer program: a randomised controlled trial. J Sports Sci. 2016;34(6):564-75.

50 Kim ES, Im JA, Kim KC, Park JH, Suh SH, Kang ES, et al. Improved insulin sensitivity and adiponectin level after exercise training in obese Korean youth. Obesity (Silver Spring). 2007 Dec;15(12):3023-30.

51 Ouchi N, Parker JL, Lugus JJ, Walsh K. Adipokines in inflammation and metabolic disease. Nat Rev Immunol. 2011 Feb;11(2):85-97.

52 Jiao P, Chen Q, Shah S, Du J, Tao B, Tzameli I, et al. Obesity-related upregulation of monocyte chemotactic factors in adipocytes: involvement of nuclear factor-kappaB and c-Jun NH2-terminal kinase pathways. Diabetes. 2009 Jan;58(1):104-15.

53 Yudkin JS, Kumari M, Humphries SE, Mohamed-Ali V. Inflammation, obesity, stress and coronary heart disease: is interleukin-6 the link? Atherosclerosis. 2000 Feb;148(2):209-14.

54 Forsythe LK, Wallace JM, Livingstone MB. Obesity and inflammation: the effects of weight loss. Nutr Res Rev. 2008 Dec;21(2):117-33.

55 Kawanishi N, Yano H, Yokogawa Y, Suzuki K. Exercise training inhibits inflammation in adipose tissue via both suppression of macrophage infiltration and acceleration of phenotypic switching from M1 to M2 macrophages in high-fat-diet-induced obese mice. Exerc Immunol Rev. 2010;16:105-18.

56 Pedersen BK, Edward F. Adolph distinguished lecture: muscle as an endocrine organ - IL- 6 and other myokines. J Appl Physiol. 2009;107(4):1006-14.

57 Izadpanah A, Barnard RJ, Almeda AJ, Baldwin GC, Bridges SA, Shellman ER, et al. A short-term diet and exercise intervention ameliorates inflammation and markers of metabolic health in overweight/obese children. Am J Physiol Endocrinol Metab. 2012 Aug;303(4):E542-50.

58 Gallistl S, Sudi KM, Aigner R, Borkenstein M. Changes in serum interleukin-6 concentrations in obese children and adolescents during a weight reduction program. Int J Obes Relat Metab Disord. 2001 Nov;25(11):1640-3.

59 García-Calzón S, Moleres A, Gómez-Martinez S, Diaz LE, Bueno G, Campoy C, et al.; EVASYON study group. Association of telomere length with IL-6 levels during an obesity treatment in adolescents: interaction with the174G/C polymorphism in the IL-6gene. Pediatr Obes. 2017 Jun;12(3):257-63.

60 Romeo J, Martinez-Gomez D, Diaz LE, Gómez-Martinez S, Marti A, Martin-Matillas M, et al.; EVASYON Study Group. Changes in cardiometabolic risk factors, appetite-controlling hormones and cytokines after a treatment program in overweight adolescents: preliminary findings from the EVASYON study. Pediatr Diabetes. 2011 Jun;12(4 Pt 2):372-80. 